\section{Synthesis and Antiangiogenic Activity of Staurosporine Derivatives}

Sir:

Staurosporine (1), a natural product first isolated by ŌMURA et al. ${ }^{1)}$ from Saccharothrix aerocolonigenes subsp. staurosporeus AM-2282 2 , was found to be a very potent inhibitor of protein kinase C (PKC) by TAMAOKI et $a l^{3)}$. Subsequently, 1 was found to have various pharmacological effects such as inhibition of platelet aggregation ${ }^{4)}$, smooth muscle concentration ${ }^{5}$, and angiogenesis ${ }^{6)}$.

We tried to develop staurosporine analogs for new and novel types of antiangiogenic agents. Thus, we focused on chemical modification of the amino sugar moiety.

This report describes the synthesis and antiangiogenic activity of staurosporine derivatives with modified amino sugar moieties.

The synthesis of a $3^{\prime}, 4^{\prime}$-olefin (4) derivative was accomplished by reductive $N$-methylation (aq. $\mathrm{HCHO}$, $\mathrm{NaBH}_{3} \mathrm{CN}$ ) and $N$-oxidation ( $m$-CPBA, $\mathrm{CHCl}_{3}$ ) followed by Hofmann degradation $\left(160^{\circ} \mathrm{C}, 1 \mathrm{mmHg}\right)^{7)}$ with a $78 \%$ overall yield in 3 steps, as outlined in Scheme 1. Next, dihydroxylation $\left(\mathrm{OsO}_{4}, \mathrm{NMO}\right)$ of $\mathbf{4}$ produced $\mathbf{5}$, which was identical to MLR-52 $2^{8}$, with a $65 \%$ yield. Protection of 5 with 1, 1'-thiocarbonyldiimidazole in THF resulted a 4',5'$O$-cyclo-thiocarbonate (6) with a $60 \%$ yield. Selective reduction $\left(n-\mathrm{Bu}_{3} \mathrm{SnH}, \mathrm{AIBN}, 47 \% \text { yield) }\right)^{9)}$ of 6 to a $4^{\prime}-(S)$ hydroxy derivative (7) followed by Moffatt oxidation ${ }^{10)}$ to ketone (8) (80\% yield) and stereoselective reduction (Kselectride, $50 \%$ yield) produced a $4^{\prime}-(R)$-hydroxy derivative (9), which was identical to RK-286 ${ }^{11)}$ (Scheme 2).

On the other hand, hydroboration of 4 [1) $\mathrm{BH}_{3}$ 2) $\mathrm{H}_{2} \mathrm{O}_{2}$ $\mathrm{NaOH}]$ gave the $5^{\prime}-(R)$-hydroxy intermediate $(\mathbf{1 0})$ with a $80 \%$ yield. Moffatt oxidation ${ }^{10)}$ of 10 to 5 -ketone (11) $(88 \%$ yield) and stereoselective reduction (K-selectride, $61 \%$ yield) produced a $5^{\prime}-(S)$-hydroxy derivative (12). Protection of 10 with 1,1'-thiocarbonyldiimidazole in THF resulted 13 with a $58 \%$ yield. Reduction of 13 with $n$ $\mathrm{Bu}_{3} \mathrm{SnH}$ and AIBN produced a $4^{\prime}$-de- $N$-methylamino derivative (14) with a $63 \%$ yield (Scheme 3 ). The structures of these derivatives were determined by NMR analysis, IR, and mass spectrometry.

Antiangiogenic activities of these compounds were investigated by the sandwich method ${ }^{12)}$. Calf pulmonary artery endothelial cells (CPAE) were suspended in a collagen gel mixture of 8 vol. Cellgen (Type I collagen, Koken), 1 vol. reconstructive buffer containing $50 \mathrm{~mm}$ $\mathrm{NaOH}, 260 \mathrm{~mm} \mathrm{NaHCO}$, and $200 \mathrm{~mm}$ HEPES, and 1 vol.

Scheme 1.

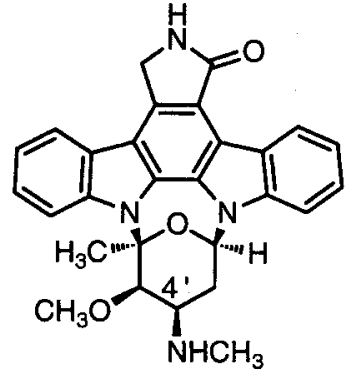

Staurosporine 1

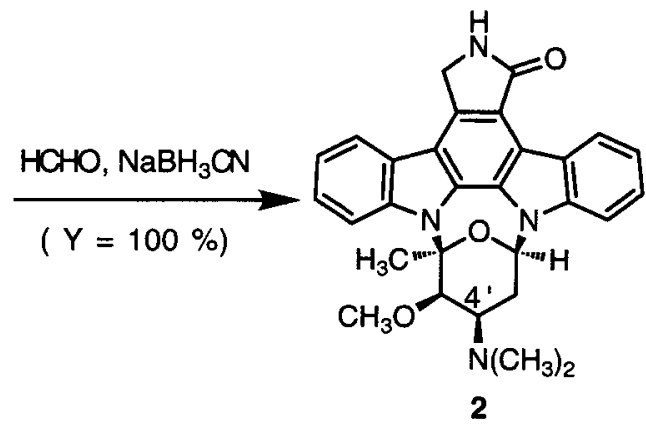

vacuum, heat

$(Y=85 \%)$

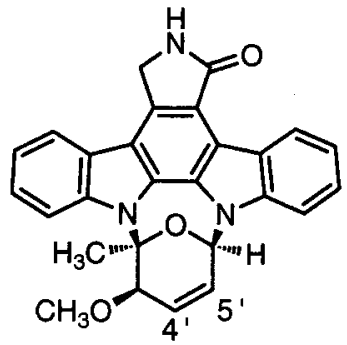

Key intermediate 4 (total yield $78 \%$ in 3 steps) 
Scheme 2.
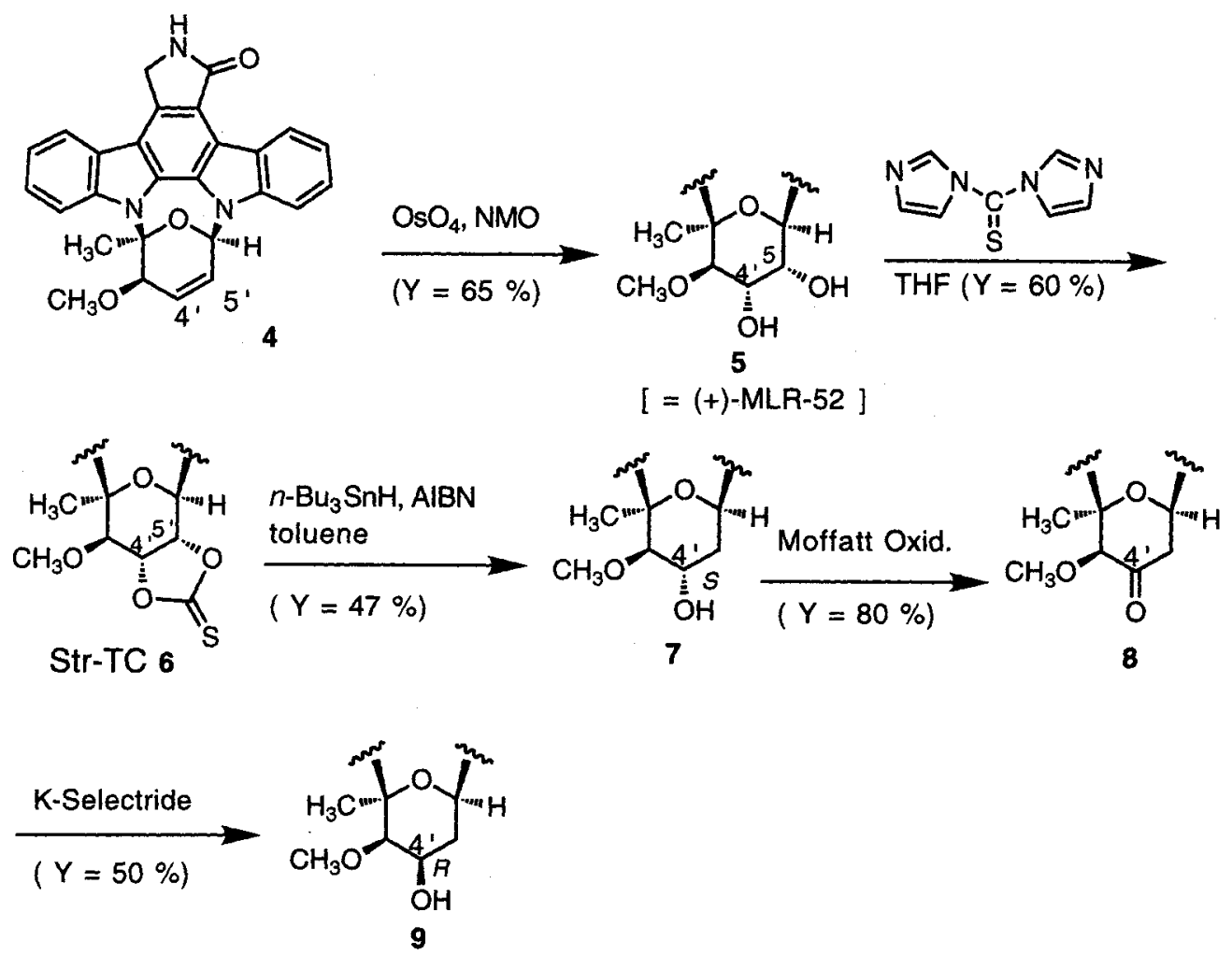

$[=(+)-R K 286 c]$

Scheme 3.

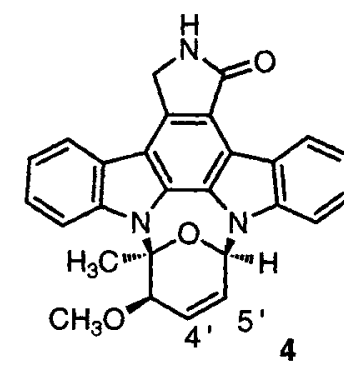

Moffatt Oxid. ( $Y=88 \%)$
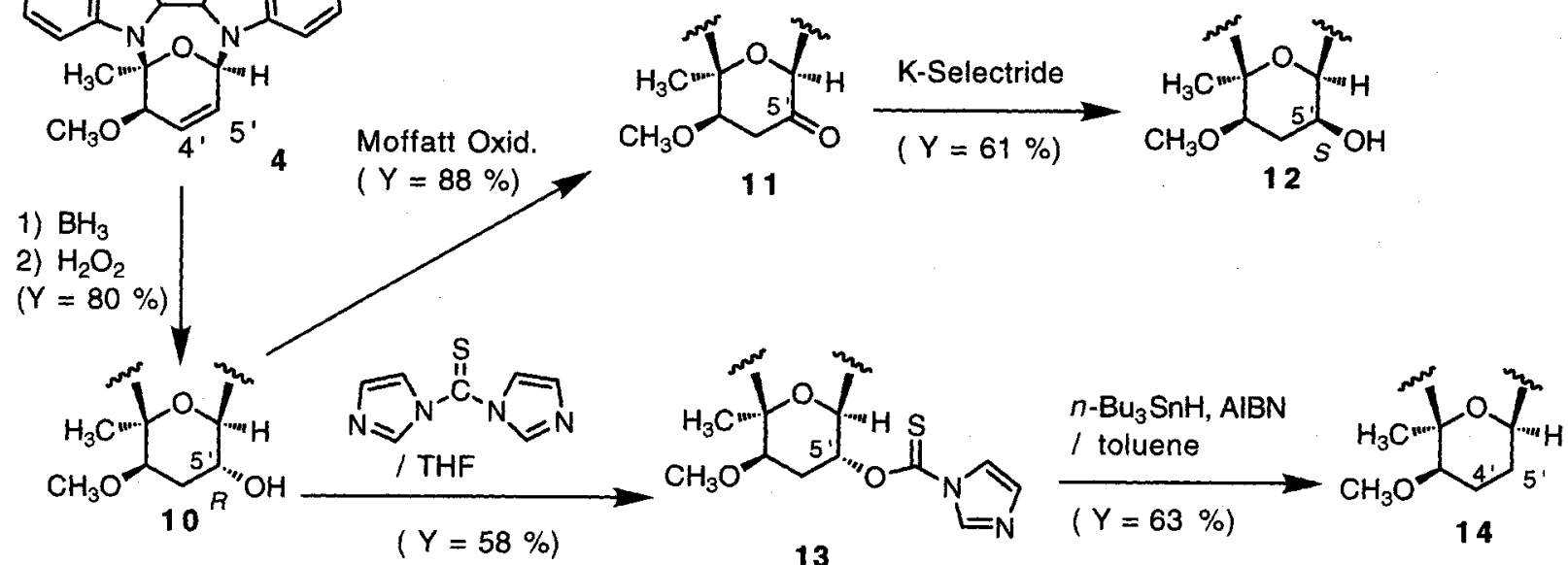
Table 1. Antiangiogenic effect of staurosporine derivatives.

\begin{tabular}{|c|c|c|c|c|c|c|}
\hline \multirow{2}{*}{ Compound } & \multirow{2}{*}{\multicolumn{2}{|c|}{$\begin{array}{l}\text { Antiangiogenesis } \\
\text { MIC }: \mu \mathrm{M}[\mathrm{A}]\left(\mathrm{IC}_{50}: \mu \mathrm{M}\right)\end{array}$}} & \multirow{2}{*}{\multicolumn{2}{|c|}{$\begin{array}{c}\text { Cytotoxicity }\left(\mathrm{IC}_{50}: \mu \mathrm{M}\right) \\
\text { CPAE }[\mathrm{B}] \text { HeLa }[\mathrm{C}]\end{array}$}} & \multicolumn{2}{|c|}{ Specificity $^{a}$} \\
\hline & & & & & {$[\mathrm{B}] /[\mathrm{A}]$} & {$[\mathrm{B}] /[\mathrm{C}]$} \\
\hline 1 & $<0.0013$ & $(0.006)$ & 0.0011 & 0.0084 & $>0.79$ & 0.13 \\
\hline 2 & $>2.1$ & & $>2.1$ & 0.055 & - & $>38$ \\
\hline 3 & 0.32 & & 1.6 & 7.3 & 5.0 & 0.22 \\
\hline 4 & $>2.3$ & & 0.30 & 5.6 & $<0.13$ & 0.053 \\
\hline 5 & 0.043 & & $>0.21$ & 0.019 & $>5.0$ & $>11$ \\
\hline 6 & 0.016 & $(0.42)$ & 2.0 & 0.35 & 125 & 5.7 \\
\hline 7 & 0.09 & & 1.1 & 1.0 & 12 & 1.0 \\
\hline 8 & 5.5 & & 3.6 & 4.0 & 0.66 & 0.91 \\
\hline 9 & 0.18 & & 0.62 & 0.26 & 3.5 & 2.4 \\
\hline 10 & 0.028 & & 0.22 & 0.027 & 8.0 & 8.2 \\
\hline 11 & 0.055 & & 0.055 & 0.27 & 1.0 & 0.20 \\
\hline 12 & 0.11 & & 0.22 & 0.073 & 2.0 & 3.0 \\
\hline 13 & $<0.04$ & $(0.22)$ & 3.0 & 1.4 & $>85$ & 2.1 \\
\hline 14 & 0.73 & & 16 & 1.1 & 22 & 15 \\
\hline
\end{tabular}

a B/A level was specific for the activity (A: Minimum Inhibitory concentration, B: cytotoxicity)

10-fold concentration of EAGLE's minimum essential medium (MEM, Gibco) at $\mathrm{pH} 7.4$, and adjusted to $2.5 \times 10^{5}$ cells $/ \mathrm{ml}$. One hundred $\mu$ l of collagen-cell mixtures was poured into a 96-well multiple well plate (Sumiron, Sumitomo) and incubated at $37^{\circ} \mathrm{C}$ in a $\mathrm{CO}_{2}$ incubator for 30 minutes. After gelation, $100 \mu 1$ of $20 \%$ FBS-MEM medium with sample was challenged and incubated at $37^{\circ} \mathrm{C}$ in a $\mathrm{CO}_{2}$ incubator for 3 days. Angiostatic activity was evaluated by the observation of the morphological change. The results are summarized in Table 1.

Staurosporine (1) showed strong antiangiogenic activity, $\mathrm{IC}_{50}=6 \mathrm{nM}$; however, it also showed strong non-specific cytotoxicity. It doesn't show selective toxicity.

The derivatives prepared using the above methodology gave decreased antiangiogenic activity, but some of them had significantly decreased cytotoxicity, and showed prominent selective toxicity. Derivatives 6, 7, 10, 13, and 14 showed high specificity (B/A) levels. 6 and 13 showed especially strong antiangiogenic activity, $\mathrm{IC}_{50}=0.42$ and $0.22 \mu \mathrm{M}$, respectively. The cytotoxicity was very weak, $\mathrm{IC}_{50}=2.0$ and $3.0 \mu \mathrm{M}$, respectively. The specificity (B/A) level was increased in both cases.

Moreover, 13 inhibited the tumor angiogenesis caused by tumor inoculation in syngenic mice in vivo. Therefore, the most interesting compound is 13. Analytical data of 13: HR-MS calcd. for $\mathrm{C}_{31} \mathrm{H}_{25} \mathrm{~N}_{5} \mathrm{O}_{4} \mathrm{~S} 563.1627$, found 563.1631;
IR (KBr) cm $\mathrm{cm}^{-1} 3128,2939,1679,1635 ;{ }^{1} \mathrm{H}$ NMR (400 $\left.\mathrm{MHz}, \mathrm{CD}_{3} \mathrm{OD}\right) \delta 1.91\left(1 \mathrm{H}, \mathrm{dd}, J=2.0,15.0 \mathrm{~Hz}, 4^{\prime}-\mathrm{H}\right), 2.39$ $\left(3 \mathrm{H}, \mathrm{s}, 2^{\prime}-\mathrm{CH}_{3}\right), 2.32 \sim 2.46\left(1 \mathrm{H}, \mathrm{m}, 4^{\prime}-\mathrm{H}\right), 3.84(3 \mathrm{H}, \mathrm{s}$, $\left.\mathrm{OCH}_{3}\right), 4.08\left(1 \mathrm{H}, \mathrm{dd}, J=3.6,12.2 \mathrm{~Hz}, 3^{\prime}-\mathrm{H}\right), 4.93(2 \mathrm{H}, \mathrm{s}, 7-$ $\left.\mathrm{H}_{2}\right), 6.21\left(1 \mathrm{H}, \mathrm{s}, 5^{\prime}-\mathrm{H}\right), 6.51\left(1 \mathrm{H}, \mathrm{s}, 6^{\prime}-\mathrm{H}\right), 7.11(1 \mathrm{H}, \mathrm{s}$, imidazole), 7.26 7.59 $(5 \mathrm{H}, \mathrm{m}$, aromatic), $7.76(1 \mathrm{H}, \mathrm{s}$, imidazole), $7.86(2 \mathrm{H}, \mathrm{m}$, aromatic), 8.48 ( $1 \mathrm{H}, \mathrm{s}$, imidazole), $9.30(1 \mathrm{H}, \mathrm{d}, J=7.9 \mathrm{~Hz}$, aromatic).

Therefore, we are very interested in testing the new staurosporine derivative $\mathbf{1 3}$ as an antiangiogenic agent.

Further details of its mode of action will be reported elsewhere.

ZHUORONG LI

TOSHIAKI SUNAZUKA

RINTARO YAMADA

YUMIKO KATO

AKIKO ENOMOTO

MASAHIKO HAYASHI

YOSHIHIRO HARIGAYA

SATOSHI OMURA*

Research Center for Biological Function, The Kitasato Institute, and Kitasato University, 5-9-1 Shirokane, Minato-ku, Tokyo 108, Japan

(Received December 9, 1999) 


\section{References}

1) Ōmura, S.; Y. Iwai, A. Hirano, A. Nakagawa, J. AWAYA, H. TSUChIYA, Y. TAKahashi \& R. Masuma: A new alkaloid AM-2282 of streptomyces origin taxonomy, fermentation, isolation and preliminary characterization. J. Antibiotics 30: 275 282, 1977

2) Takahashi, Y; M. Shinose, A. Seino, Y. Iwai \& S. OMURA: Transfer of saturosporine-producing strain Streptomuces staurosporeus AM-2282 to the genus Saccharothrix aerocolonigenes. Actinomycetologica 9: 19 26, 1995

3) Tamaoki, T.; H. Nomoto, I. Takahashi, Y. Kato, M. MoRimoto \& F. Tомта: Staurosporine, a potent inhibitor of phospholipid/ $\mathrm{Ca}^{++}$dependent protein kinase. Biochem. Biophys. Res. Commun. 135: 397 402, 1986

4) Ōmura, S.; Y. SASaKi, Y. IWAi \& H. TAKeshima: Staurosporine, a potentially important gift from a microoraganism. J. Antibiotics 48: 535 548, 1995

5) Seto, M.; Y. Sasaki \& Y. SAsaki: Alternation in the myosin phosphorylation pattern of smooth muscle by phorbol ester. Am. J. Physiol. 259: C769 C774, 1990

6) Oikawa, T.; M. Shimamura \& H. Ashino: Inhibition of angiogenesis by staurosporine, a potent protein kinase inhibitor. J. Antibiotics 45: 1155 1160, 1992

7) JoNEs, P. A. \& E. K. Rowley: Chemical modification of erythromycin antibiotics, I. 3'-De(dimethylamino)erythromycin A and B. J. Org. Chem. 33: 665 670, 1968

8) McAlpine, J. B.; J. P. Karwowski, M. Jackson, M. M. Mullally, J. E. Hochlowski, U. Premachandran \& N. S. BuRRES: MLR-52, (4'-Demethylamino-4',5'-dihydroxystaurosporine). A new inhibitor of protein kinase $\mathrm{C}$ with immunosuppressive activity. J. Antibiotics 47: $281 \sim 288,1994$

9) Lartey, P. A.; R. Faghih, T. Pagano, H. N. Nellans, A. Patersen \& J. J. Plattner: Stereoselective deoxygenation of erythromycin A at C-12: Effect of structure and conformation on prokinetic activity. J. Antibiotics 48: $730 \sim 732,1995$

10) Nagamitsu, T.; T. Sunazuka, H. Tanaka, S. Ōmura, P. A. Sprengeler \& A. B. SMith, III: Total synthesis of (+)-lactacystin. J. Am. Chem. Soc. 118: 3584 3590, 1996

11) Osada, H. ; H. Takahashi, K. Tsunoda, H. Kusakabe \& K. IsONO: A new inhibitor of protein kinase C. RK286c (4'-demethylamino-4' -hydroxystaurosporine). I. Screening, taxonomy, fermentation and biological activity. J. Antibiotics 43: 163 167, 1990

12) Kanayasu, T.; J. NAKANO-HaYAShI, N. Asuwa, I. Morita, T. Ishi, H. ITO \& S. MAROTA: Leukotoriene C4 stimulates angiogenesis in bovine carotid artery endothelial cells in vitro. Biochem. Biophys. Res. Comm. 159: 572 578, 1989 